Aus dem Elisabeth-Krankenhaus Kassel.

\title{
Der Luftkompressor im Krankenhaus ${ }^{1}$ ).
}

\author{
Von Dr. Franz Kuhn.
}

II. Teil.

Die Hochdruckmassage.

Allgemeiner Teil.

Dic: Literatur der letzten Jahre ist beherrscht von den Fragen der Hyperämie in ihrer Anwendung als Heilmittel. Die Einzelmitteilungen über diese Dinge zählen nach Hunderten, die Versuche und Finzelbcobachtungen sind unzählbar.

In seinem grundlegenden Werke hat $\mathrm{Bier}$ neben der Stauungshyperämie die große Bedeutung der aktiven oder Fluxionshyperämie für die Therapie dargelegt, und ihre großen physiologischen und klinischen Wirkungen betont.

Für diese letztere nun hat er als souveränes und wesentliches Mittel die $\mathrm{H}$ e i B l uft erkannt und von ihr ausgiebigen Gebrauch gemacht und zu machen gelehrt.

Wenn er auch andererseits bereits die Esmarchsche Blutlecre und die Hofmeistersche Quecksilbermassage als ähnliche Mittel zur Erzeugung von aktiver Hyperämic anführt, so haben diese doch bis zur Stunde eine untergeordnete Stelle behalten.

Jedenfalls sehr intensiv weiter verfolgt wurden diese Arten von Hypcrämie seitdem nicht.

Vielleicht sind es äußere Gründe, daß diese Art von Blutbewegung bis jetzt nicht weiter geprüft und erörtert und dementsprechend ausgebaut wurde, vielleicht auch technische und konstruktive.

Nachdem ich nun aber gesehen, welch mächtiges mechanisches Mittel wir den Organen des Körpers gegenüber in dem

I) Vgl. meine seitherigen Aufsätze in dieser Zeitschrift und der Deutsch. med. Wochenschrift. 
L uf t d u ck haben, und nachdem ich gesehen, wie relativ einfach es andererseits ist, und wie schonend es bei aller Kraftleistung und Kraftentfaltung sich machen läßt, bei Anwendung von Luftdruck große Druckwirkungen auf einzelne Organe und Organtcile auszuüben 1 ), und wie bequem es ist, mittels solchen Luftdruckes bald absolute Blutlecre, bald die entgegengesetzte Hyperämie zu schaffen, mußte es nahe liegen, genauer die physiologischen und klinischen Einzelheiten dieser Dinge experimentell zu prüfen.

Dies um so mehr, als der Luftdruck ja als solcher nicht ctwas ganz Neues in der Medizin ist, und bereits einige Luftdruck-Vorarbeiten, wenn man es so nennen will, aus früheren Zciten vorliegen. Sie weichen von unseren Versuchsanordnungen allerdings insofern ab, als sie wohl auch den Luftdruck auf den menschlichen Körper anwenden, ihn aber in umgekehrter Weise ausnutzen, nämlich als negativen Druck oder als Saugluft. Wer Orientierung über diese Dinge sucht, lese die Auseinandersetzungen, die Bier in seinem Hyperämiewerke (Seite 89-- Io9 über große Saugapparate) gibt. Die dortigen Ausführungen haben naturgemä $B$ allerlei grundlegende Bedeutung für unsere Fragen, wenn sie auch den Hochdruck nicht berühren.

Wic man dort ersieht, hat man sich nämlich in der Mitte des vorigen Jahrhunderts schon einmal in größerem Maßstabe des Luftdruckes, sowohl des positiven als auch des negativen, zu klinischen Zwecken bedient:

Junod ${ }^{2,3,4}$ ) machte in den vierziger Jahren großes Auf-

1) Auf die rein mechan isch-orthopäd ischen Seiten der betreffenden Fragen (vgl. $13 \mathrm{i} \in \mathrm{r}, \mathrm{S}$. I $(22)$ will ich an dieser stelle nicht eingehen; ich werde sie in einer besonderen Abhandlung bringen, elenso soll der $P u$ e u mat ot herapic eine cigene Abhandlung, sowohl was Cberdruck als linterdruck betrifft, gewidmet sein.

2) J unod, Recherches physiologicues et thérapeutiques sur les effets de la compression et de la raréfaction de l'air, tant sur le corps que sur les membres isolés. Revue méd. franc. et étrang. I834. Tome III, \$. 350 und Bericht der Akademie ebenda s. 460 .

3) J u nod, Note sur un nouvel appareil dit grande ventouse proprè à faire le vide sur la moitié inférieure du corps etc. Gaz. médic. de Paris is.3s. I'. VI. Nr. 25, S. 388.

4) J u nod, Couvelles observations sur l'emploi des appareils hémospasiques et des bains d'air comprimé, lues à l'académie de sciences. Paris is 43. 
sehen mit seinen Arbeiten über komprimierte und verdünnte Luit, seinen Ventousen und Stiefeln.

Auch in Deutschland fanden die Methoden Freunde und Nachahmer, wie aus den Arbeiten von Ficinus und Jourdan über die Hämospasie hervorgeht, und der Apparat von Erpenbeck beweist.

Bi er griff dann bekanntlich die Saugtherapie von ganz anderen Seiten mit anderen Absichten und Voraussetzungen und mit anderen lirfolgen an.

Uns interessieren alle diese Dinge sehr. Aber überblicken wir alle diese Arbeiten, so finden wir wenig über positiven Druck in unserem Sinne, und geradezu nichts über $\mathrm{Hochdruck}$ in unserem Sinne.

Treten wir daher im Folgenden den Fragen in letzterem Sinne näher.

\section{Abschnitt.}

Die mechanische Applikation hohen Druckes auf die menschlichen Körperorgane.

A p parate für diese $Z$ wecke.

Bevor man an die Anwendung und Ausnutzung von Hochluftdruck im klinischen Sinne denken konnte, galt es ein zweck-

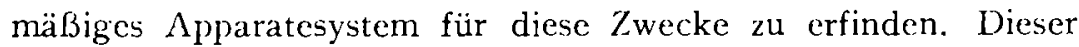
Aufgabe war nicht so leicht gerecht zu werden.

Ist es doch nicht so einfach, Luft höherer Spannung am menschlichen Körper zu applizieren: es fehlt sehr leicht an der nötigen Dichtung. Denn die Kräfte, die in Frage kommen, sind gro $B$, und de Abdichtung andem Körper is t in allgemeinen dannschwer zubewerkstelligen.

Auch die Art und Kombination des Materials und der Stoffe war schwer herauszufinden, namentlich auch deswegen, weil große Dichtigkeit neben großer Haltbarkeit in Frage steht.

Gummi allein wäre für diese Zwecke viel zu dehnbar und viel zu schwach. Die Stoffe der Äroplane sind viel zu undicht, um Verwendung zu finden. $\Lambda \mathrm{m}$ besten eignen sich gute Luftballonstoffe oder die Paragummistoffe bester Qualität unserer Operationswäsche. 
Aber auch diese können leicht zu schwach sein und bedürfen schützender Verstärkung. Genäht dürfen die Hüllen nie werden. Die Naht ist zu undicht. Hier darf nur geklebt werden.

Die Art der Applikation kann mannigfach sein: zu unterscheiden sind von 'vornherein verschiedene Arten von Applikationen, je nachdem man ein Glied oder Organ t o tal-zirkulär oder p a r ti ell-zirkulär umschnüren will, oder je nachdem man bloß von einem festen Widerlager aus einen Druck gegen ein Organ auszuüben die Absicht hat.

Nach diesen drei prinzipiellen Gesichtspunkten sind daher auch die Apparate zu unterscheiden, die wir im Folgenden zeigen wollen.

Diese Einteilung fällt in vielen Punkten zusammen mit den verschiedenen Spezialarten von klinischen Anwendungen.

Nach den genannten Prinzipien hätten wir also vorerst drei Typen von Weichstoffhüllen für Luftdruck für die ins Auge genommenen Zwecke zu unterscheiden.

a) Stulpen- und manschettenförmige Apparate.

Zunächst kommen zwei Arten von Stoffhüllen in Betracht, entweder eine Doppelstulpe oder eine Doppelmanschette. Hat man dünnere Organe, z. B. eine Extremität to tal zu umschnüren, so wählt man einen die ganze Extremität übergreifenden Doppelsack oder anders gesagt eine Doppelstulpe, in deren Mittelraum die Druckluft zirkuliert und in deren Innenraum das betreffende Organ hineingesteckt werden kann.

Für mehr örtliche Verwendungen, z. B. auch am Knie oder Ellenbogen, eignet sich besser die doppelwandige Hohlmanschette.

Für diese letzteren partiellen Anwendungen z. B. am Knie, Ellenbogen usw. kann man sich allerdings auch der alsbald zu schildernden schlauchförmigen Einfachluftsack b inde, die in einen stärkeren Segeltuchbezug eingebunden ist und mittels dieses Bezuges auch über dem Organe befestigt werden kann (vgl. das folgende Kapitel), mit Vorteil bedienen.

Was Einzelheiten unserer Apparate betrifft, wäre noch folgendes zu bemerken: 


\section{Die Stulpe}

ist ein in sich eingestülptes flaschenförmiges oder strumpfartiges, kegelförmiges Gebilde. Dic eingestülpte Innenkegelfläche muß nahe der Eintrittsstelle der Luft an dem Eintrittsrohre befestigt sein. Sonst wird sie beim Aufblasen herausgedrückt.

Die Manschette.

Die Manschette ist, wie der Muff unserer Damen, ein doppelzylinderisches, an beiden Enden geschlossenes Hohlgebilde, das demnach eine Außen- und eine Innenwand hat, in deren Mittelraum die Kompressionsluft eingefüllt wird.

\section{Die Überzüge.}

In beiden Anwendungen, ob Stulpe oder Manschette, legt sich bei der Aufblasung die Innenwand dem fraglichen innen hineingesteckten Organteile oder Gebilde, den es umschnürt, dichte an, und vermittelt ihm direkt den Druck, den man in den Raum zwischen Außen- und Innenwand einpreßt.

Das äußere Blatt hebt sich, soweit es ihm seine Form und Größc, besser noch, soweit es ihm ein übergezogener derberer Segeltuchbezug oder ein Blechzylinder gestattet, nach auben unter intensiver Spannung ab.

Was diese stärkeren $\ddot{U}$ ber $z$ üg e betrifft, noch einige Bemerkungen :

Es können weiche und starre I3ezüge zweckmäßig sein. Die erstcren bestehen am besten aus einem genähten oder schnürbaren Futterale aus Segeltuch, ähnlich wie die Reisebezüge einer Reisedecke oder eines Regenschirmes, die Rohre aus Blech können zylindrisch oder konisch oder stiefelförmig sein.

I3eide Arten von Bezügen werden vor der Verwendung des Luftdruckdoppelsackes über denselben gezogen; sie halten dann leicht den Atmosphärendruck bei der Aufblähung aus.

b) Bindeförmige Luftsackapparate.

Diese Form der Ausführung ist von allen vielleicht die nächstliegende. Sie nähert sich, grob gesagt, in der äußeren Form 
einer Schwimmblase oder einem Schwimmgürtel. Ihre Anwendung erfolgt sowohl auf Massivorgane (Extremitäten usw.) als auch auf Hohlorgane.

Der Grundtypus einer Luftsackbinde der beschriebenen Art setzt sich zusammen aus einem mehr oder minder umfangreichen, schlauchartigen, auf beiden Seiten geschlossenen, länglichen Luftsackgebilde, das in eine stärkere Umhüllung eingebunden wird. Durch eine zentrale Zuführung steht der Luftsack mit der Luftdruckleitung in Verbindung. Mittels des Segeltuchbezuges wird der Luftsack (dann natürlich in luftleerem Zustande) auf den betr. Organteil aufgebunden. Dabei ist es wünschenswert, daß der Scgeltuchbezug im Interesse der Festigkeit bei der nachfolgenden Luftdruckanwendung den Organteil sowohl wie den Luftsack mehr als einmal tunlichst exakt und geschlossen umkreist und ihn zuverlässigst umschließt. Denn die in Anwendung kommende Kraft ist gewaltig.

Naturgemäß haben die Binden sowohl, wie ihre Luftsackeinlagen verschicdene Längen und Breiten und somit auch die aufgeblasenen Säcke verschiedenes Kaliber.

Ich gedenke bei den Einzelverwendungen im speziellen Teil auf die verschiedenen technischen und konstruktiven Einzelheiten zurückzukommen. Der wichtigste Punkt ist cine gewisse Widerstandsfähigkeit des ganzen Apparates und eine sehr zuverlässige Anlegung, da die Kräfte, die in $1 / 2^{-}$I $\Lambda$ tmosphäre zur Geltung kommen; doch recht respektabel sind.

c) Pelottenartige Druckluftapparate mit starrem Wid erlager.

Diese Apparate ermöglichen eine mehr zirkumskripte pelottenartige Druckluftanwendung, charakterisiert durch die IJruckwirkung eines relativ großen Pelottekissens, das mittels eines starren Gestelles u. dgl. gegen das zu massierende oder zu komprimierende Organ gehalten und gepreßt wird.

Der ganze für diese Frage in Anwendung stehende Apparat besteht sonach 1. aus irgendeiner Form eines starren Gestelles (hufeisen-, korsett-, tonnenförmig), das mehr oder minder um den zur Behandlung kommenden Organteil greifend, einen festen Punkt abgibt, von dem aus dann die Pelotte auf den betreffenden 
Körperteil (natürlich nachdem zuvor der Körper im Innern des Gestelles fixiert ist) wirken kann. 2. Aus einer Luftsackpelotte von vcrschiedener Form, Größe und Ausdehnungsfähigkeit, welche zwischen dem genannten festen Punkt und dem Organteile zur Aufblasung kommt.

d) Die Lieferung des Luftdruckes.

Der Bezug der Druckluft erfolgt nach unserer Anordnung am besten aus den Verteilungsanschlüssen unserer Luftdruckleitungen (vgl. $\mathrm{Kuhn}$ : Der Luftkompressor im Krankenhaus ${ }^{1}$ )), doch können im Notfalle auch Bomben herangezogen werden.

Die erstere Anlage ist aber ausgiebiger und erlaubt mit mehr Verschwendung zu arbeiten.

Dic Messung des Druckes erfolgt durch ein vorgeschaltetes Manometer.

Die Hähne erlauben die feinste Einstellung und jede beliebigc Variation.

\section{Abschnitt.}

\section{Die Wirkungen des hohen Luftruckes am und auf den mensch- lichen Körper.}

Dic Wirkungen eines auf die Körperorgane angewandten Luftdruckes sind, wie leicht begreiflich, zweicrlei:

I. zunächst kommen $\mathrm{mech}$ a $\mathrm{n}$ is $\mathrm{ch}$ e Wirkungen in Frage, ebenso mechanisch wie der Zug und I)ruck einer Schraube oder eines Gewichtes,

2. dann sind aber spezifisch physiologis che Wirkungen in Betracht zu zichen.

I. Mechanische Wirkungen hohen Luftdruckes am menschlichen Körper.

a) Massivorgane.

Dic mechanischen Wirkungen hohen Luftdruckes auf einzelne Organe des menschlichen Organismus sind zweierlei, je

1) Vgl. die früheren Arbeiten in dieser Zeitschrift, Bd. I04 und in der Deutsch. med. Wochenschrift 1900, Nr. 45; 1909, Nr. 50; 1910, Nr. 3 I. 
nachdem sie die Form und Gestalt der betreffenden Organe oder ihren Inhalt betreffen.

Gestalt und Form des Skelettes zu beeinflussen stehen zurzeit Druck und Zug von Schrauben und Gewichten in Anwendung, sei es, daß ein verbogener Knochen oder ein asymmetrischer Thorax in Frage steht.

Es ist begreiflich, daß für eben diese Zwecke mit Vorteil auch der Luftdruck herangezogen wird, um so mehr, als mit seiner Hilfe sehr hoher Druck in sehr schonender Form zur Geltung gebracht werden kann. Luftkissen als Druckpelotten sowohl denn als Gegendruckpolsterkissen bewähren sich bekanntlich sehr gut.

Kein Wunder, daß auch bereits Vorschläge und kleinere Versuche nach dieser Richtung vorliegen. Im größeren Stile fehlt noch eine rationelle Ausbeute des Luftdruckes für orthopädische Zwecke. Ich werde in einer Spezialabhandlung darauf zurückkommen.

Andeutungsweise seien einige Gebiete gestreift. Da ist es die Polsterung von Körperteilen bei Zug und Gegenzug mittels Luftdruckkissen; dann die Skoliosen- und Kyphosenbehandlung, wo der Luftdruck mit Vorteil Verwendung findet, dann die Nachbehandlung von Frakturen usw. Ferner die Beweglichmachung von Gelenken (wie ja auch schon Bi er seine Luftleere zu diesem Zwecke gebraucht), endlich jede Art von Massage.

Die Orthopädie wird somit zweifellos in ungemein vielseitiger Wcise den Luftdruck gebrauchen können.

Die anderen medizinischen Disziplinen werden in anderer Weise den Luftdruck in seiner Wirkung auf Massivorgane ausnutzen, sie werden ihn gebratuchen, insofern als die Blut- und Zirkulations- und Stoffwechselverhältnisse in denselben in Frage sind. Diese Art der Anwendung hat dann mehr physiologisches Interesse, und werden wir diese Dinge unter den physiologischen Wirkungen bringen (Seite 206).

b) Hohlorgane.

Ebenso wie auf Massivorgane sind naturgemäß die Luftdruckanwendungen mechanischer Art auf Hohlorgane von klinischer Bedeutung. Es wird kaum eine zartere und dabei doch so aus- 
giebige Kompression einer Gallenblase oder Harnblase, ferner eines Magens usw. oder Darmes usw. oder eines Thorax möglich sein, sei es in toto oder partiell, als mittels unserer Luftdruckapparate.

Gehen wir die Einzelzwecke nur andeutungsweise und in Kürze durch, so wird sich dies alsbald bestätigen. Eine rationelle Dickdarmmassage läßt sich gewiß auf keinem Wege so intensiv und doch so schonend durchführen, wie mittels Luftdruckes (siehe hierzu den speziellen Teil).

Dasselbe gilt von gewissen Formen von Massage des Magens. Ein neues, sicher recht segensreiches Gebiet ist eine rationelle Massage der Hypochondrien sowohl für Fälle von träger Zirkulation in den Gallenwegen als solcher im Dickdarm.

Auch der Thorax zieht von der mechanischen Leistung des Luftdruckes großen Nutzen:

Zunächst sind es die Asthmatiker, die von der mechanischen Bceinflussung der Thoraxwände profitieren, wenn wir einen Atmungsstuhl, der mittels Luftdruckes arbeitet, vorlegen (vgl. meine Artikel über Asthma und Pneumatotherapie).

Ebenso sind es Herzleidende, die durch Luftdruck Erleichterung finden.

Auch die Orthopädie des Thorax hat bereits, wie oben berührt, den Luftdruck sich zu Nutzen gemacht (siehe später).

II. Physiologische Wirkungen des hohen Druckes auf die menschlichen Körperorgane.

Bei der Analysierung der physiologischen Wirkungen unseres Hochluftdruckes, wobei zunächst Massivorgane in Frage stehen, möchte ich in der Hauptsache der Bierschen Finteilung, wie diescr sie für seine Hyperämie gegeben hat, folgen, und mir die Fragen vorlegen:

Wic weit wirkt die Hochdruckanämie und Hyperämie

I. anästhesierend und schmerzlindernd,

2. resorbierend und auflösend,

3. plastisch neu bildend. 
a) Die anästhesierende Wirkung.

$\mathrm{B}$ i e r ist der Ansicht, daß die Herabsetzung der Empfindlichkeit eine allgemeine Eigenschaft der Hyperämie ist. $\mathrm{Ob}$ dabei die Entfernung der Krankheitsursache durch die stärkere Blutdurchströmung mit im Spiele ist, oder ob nach $\mathrm{R}$ i t t e $\mathrm{r}$ die seröse Durchtränkung des Gewebes bei der Stauung die Empfindlichkeit der Nerven herabsetzt, bleibt noch dahingestellt.

$\mathrm{R}$ indfleisch beobachtete an sich, was seine gichtigen Gelenkschmerzen betraf, eine wesentliche Linderung durch die Quecksilbermassage.

Es bedarf noch der Kontrolle an einem guten klinischen Material, in welchem Maße und unter welchen Voraussetzungen unsere Hochdruckmassage anästhesierend wirkt. Dann lassen sich vielleicht auch weitere Hypothesen zu ihrer Erklärung aufstellen.

Daß nicht die Anämie oder „Dekongestionierung“ schmerzstillend wirkt, wissen wir seit Bi er genugsam.

b) Dic resorbierende Wirkung.

Es kann von vornherein keinem $Z$ weifel unterliegen, dal3 wir bezüglich der Resorption in unserem Hochdruck eines unserer kräftigsten Hilfsmittel haben, sei es, daß er in manchen Fällen allein zur Anwendung kommt, sei es, da $B$ er im Zusammenhang und in Zusammenwirkung mit anderen Verfahren und Methoden der Hyperämie Ausnutzung findet.

Wenden wir auf ein zu behandelndes Organ, sei es zum Beispiel die Kallusstelle eines Knochens oder ein ödematöses Bein, den Hochdruck an, so erfolgt zweierlei :

I. eine primäre, arterielle, sich bis zur absoluten steigernde Anämie und damit eine mechanische Beseitigung aller beweglichen Dinge, also auch Ödemflüssigkeit, daneben selbstverständlich des venösen Blutes, der Lymphe, der parenchymatösen Zerfallsprodukte auf jedem verfügbaren Abfuhrwege.

2. eine sekundäre (der Anämie folgende) Hyperämie, durch verstärkte Fluxion ähnlich wie bei der Anwendung von Heißluft.

Wenn wir nun nach den Forschungen (Orlow, Heidenhain, Hamburger, Ascher, Munk, Klapp) der letzten 
Jahre zu der Überzeugung gekommen sind, daB die ganze Resorption wässriger und wasserlöslicher Stoffe durch die Blutkapillaren erfolgt, und wir in der Hyperämie ein mächtiges Mittel zur Beschleunigung der Resorption haben, so dürfte unsere Bluthochdruckmassage unter den Mitteln zur Förderung und Erzeugung derartiger Wirkungen mit in erster Reihe stehen. Ich will versuchen, dies in folgendem $\mathrm{zu}$ beweisen.

Wir wollen an dieser Stelle nicht genauer auf die sich etwas widersprechenden älteren Versuche über akut gesetzte Anämien und die Beeinflussung der parenchymatösen ,Resorption durch solche Anämien, vor allem durch Aderlässe", eingehen. $\mathrm{Kaupp}$ und Vierordt ${ }^{1}$ ) hatten die Ansicht von Reclard ${ }^{2}$ ) bestätigt, wonach Aderlässe die Resorption und somit den Eintritt der ersten Vergiftungserscheinungen verzögern sollten.

$\mathrm{Mag}$ endie hatte das Gegenteil gefunden, wenigstens was die Resorption von der Pleurahöhle betraf.

Nach seinen Ergebnissen wurde diese durch Aderlässe vermehrt. Diese letztere Ansicht hatte dann sichtlich für die Physio. logen (Weber) Geltung behalten, und seit jener Zeit durfte es als ziemlich wahrscheinlich gelten, daß eine akute Anämie die folgende Resorption in Summa fördert.

Soweit die Verhältnisse bei der Aderlaßanämie. Wichtiger als diese Versuche, welche mit akuten, aber immerhin längerdauernden Anämien arbeiteten, sind aber für unsere Fragen Versuche mit momentanen, rasch vorübergehenden Anämien, z. B. die Versuche von Wölfler und die von Klapp:

Wölfler versuchte die Resorption zu prüfen, während und nach einer $E s m a r c h s c h e n$ Blutleere:

Die Versuche wurden so angestellt, daß er Hunden $3 \mathrm{~g}$ einer 5 prozentigen wässerigen Ferridcyankaliumlösung auf eine Wunde am Sprunggelenk aufträufelte; die gewöhnliche Zeit des Erschcinens der Reaktion im Harn stellte er auf 30 Minuten fest. Im Gegenversuche machte er dieselbe Extremität blutleer mittels einer Esmarchschen Binde und träufelte dann die

I) Vierord, Lehrbuch der Physiol, S. $4 \%$

2) Reclard, Grundril der Phys. IS6I, S. I42.

3) L, u dwig, Bd. 2, S. 565 . 
vierfache Menge, also $12 \mathrm{~g}$ Farbstofflösung auf eine gleich große Wundc. Während der Zeit der Blutleere trat keine Reaktion im Harn auf. Als nach Ablauf einer Stunde die Blutleere gelöst wurde, stellte sich bereits Io Minuten später ein deutlicher blauer Nicderschlag im Harn ein.

Einmal war also mit Sicherheit die Resorption des Farbstoffes während der Zeit der Blutleere ausgeschlossen; ferner glaubt Wölfler, die Aufnahme der eingespritzten Farbstofflösung in den Kreislauf erfolge nach Abnahme des Schlauches weitaus rascher,

Un nun dem Finwand zu begegnen, daß von dem peripher vom Schlauche gelegenen Teile der Exxtremität die aufgeträufelte Flüssigkeit aufgenommen sein könnte und nur deshalb nicht in den Organismus gelangt wäre, weil der Schlauch ein mechanisches Hindernis abgäbe, änderte er den Versuch folgendermaßen:

Nach Anlegung der Blutleere in der Inguinalgegend träufelte er wiederum Ferricyankaliumlösung auf eine Wunde. Nach 35 Minuten, während denen keine Reaktion im Harn nachweisbar war, legte er einen zweiten Schlauch etwa zwei Finger breit über der Wunde an und löste den ersten Schlauch in der Inguinalgegend. Auch jetzt trat keine Reaktion auf; erst als nach einer weiteren halben Stunde auch der zweite Schlauch abgenommen wurde, erschien 6 Minuten später die Reaktion im Harn. Die Versuche wurden wiederholt auch mit größeren Farbstoffmengen und mit Strychnin angestellt, und ergaben dieselben Resultate.

Wölfler folgert nun aus diesen Versuchen, daß während der Blutleere nicht bloß keine Flüssigkeit in die Zirkulation gelangt, sondern auch nicht in jenen Teil der Extremität, welcher sich zwischen der Wunde und dem Schlauch befindet; ferner, daß die Resorption nach Abnahme des Schlauches viel rascher erfolgt als unter normalen Verhältnissen.

Wölflerschließt somit aus seinen Versuchen, da $B$ die Hyperämie, welche der Esmarchschen Blutlecrefolgt, die Resorptionsehrbeschleunigt. Ebenso beweist $\mathrm{K} \mathrm{app}^{1}$ ) in unumstößlicher Weise an einem

I) K la p p, l'ber parenchymatöse Resorption. Archiv f. experim. Pathol. und Pharmolologie Bd. 47, S. 86. 
großen Versuchsmaterial, daß die Heißlufthyperämie die Resorption von Milchzuckerlösung, die subkutan einverleibt wird, sehr wesentlich (bis auf das Doppelte bis Mehrfache) fördert, und dies auch bei Ausschaltung aller Bewegungen (z. B. in Narkose).

Auch von der Stauungshyperämie behauptet und beweist er eine Beschleunigung der Resorption, wenn auch auf anderem Wege.

$\mathrm{K}$ i a p p, der die besten experimentellen Arbeiten zu der Resorption durch Änderung des Blutgehaltes der Gewebe geliefert, kommt am Ende seiner Arbeit zu dem Schlusse:

„Wenn es mithin für beide Formen der Hyperämie, der Heißluft- und der Stauungshyperämie erwiesen ist, daß sie resorptionsfördernd wirken, dann is t es für jede andere Form der Hyperämie ebenso anzunehmen."

Vcrgleichen wir nun mit den erstgenannten Hyperämien (durch Hitze, Aderlaß) die Blutverhältnisse bei unserer Hochdruckblutmassage und die nach den genannten Experimenten zu folgernden Resorptionsbedingungen bei unserer Massage, so müssen wir schon rein theoretisch die besten Erfolge erwarten:

Denn wir haben in unserem Falle in rasch wiederholter Folge die Esmarchsche Blutleere von Wölfler mit ihrer rein mechanischen Epressionswirkung: Dann die ihr folgende Hyperämie mit der von Wölfler schon gesehenen und von Klapp bcwiesenen Resorptionsbeschleunigung durch Hyperämie, und alles dies in fortgesetzter Wiederholung und beliebig abzustufender Aufeinanderfolge.

Wenn Kla p p meinte, ,größere Extreme (als die Stauungshyperämie und Heißlufthyperämie) gäbe es wohl kaum, so könnte man dics ebenso (natürlich wieder in anderer Weise) von unserer rasch sich folgenden Anämie und Hyperämie behaupten. Es fallen eben nur die die Hitzewirkung und andere, die Transudation und Exudation, die Diffusion und Imbibition unterstützenden Momente: weg.

Zichen wir aus diesen Tatsachen die klinisch-praktischen Konsequenzen, so sehen wir, wenn wir uns kurz fassen wollen:

I. Dic Hochdruckmassage ist diegegebene und naturgemäße Mit- und Nachbehandlung bei der Anwendung der Bierschen Hyperämie. 
Sie dient, da nach Bi er bei länger dauernden Stauungen sichtlich eine Verzögerung der Resorption gesetzt ist, zur Anfachung und Beschleunigung der letzteren und zur Beseitigung des nach der Stauung vorhandenen Ödems.

Sie vermag ferner die Wirkung der Heißluft zu unterstützen.

2. Sie ist eine neue, selbständige Behandlungs artbeiallen Formenvon Ödem derKörperorgane, z. B. bei Chlorose. Anämie, nach erschöpfenden $\mathrm{Krank}$ heiten usw.

3. Sie ist die gegebene Massagebehandlung bei allen Staungsgeschwüren am Unterschenkel, ulcus cruris, elephantiasis usw.

4. Sic ist dic beste Massagebehandlung f.ür jeden Callus und vorallem für Gelenkfrakturen.

c) Gewebsbildende, dasphysiologische Wachstum fördernde Wirkung.

C̈ber die gewebsneubildende Wirkung der Hochdruckmassage läßt sich an dieser Stelle noch nichts Abschließendes sagen.

Als unbestreitbar muß zunächst rein theoretisch die Annahme gelten, daß die rasch wechselnde Durchströmung mit Blut im ganzen ein mächtiger Faktor für die Anregung der Zellentätigkeit und des Gewebswachstums und somit der Gewebsbildung ist.

Lier sagt (Seite I6. 17: Hyperämie als Heilmittel). „Starke aktive Hyperämie wirderzeugt durch die gesteigerte Tätigkeit von Körperteilen, insbesondere der Muskeln, jede $A r t$ von Reibung, die Anwendung der Massage und der Elektrizität.

Wahrscheinlich beruht ein guter Teil dieser sehrwirksamen Heilmittelebenaufder Erzeugung dieser Hyperämie."

Gilt ein derartiger Einfluß also von jeder Massage, so muß unsere Hochdruckmassage mit ihrem mechanischen, einer erhöhten Selbstbetätigung sich nähernden, allseitig gleich wirkenden mechanischen Reiz, ihrem resorptiv-anämisierenden Einfluß und ihrer dann folgenden kongestionierenden Rückwirkung einen großen, die Gewebsbildung mächtig belebenden Faktor bilden. 
Wir kennen durch Bier die gewebsbildende Wirkung der Hyperämie. Für unseren Fall kann natürlich von einer Ähnlichkeit der Vorgänge mit jenen, wie sie Bier bei Art seiner Hyperämie, der Stauungshyperämie nach Venenthrombosen schildert, nicht die Rede sein.

Dafür vergleichen sich aber schon in mancherlei Richtung die Dinge hier mit jenen Beobachtungen, in denen Bier durch arterielle Hyperämie ein Wachstum in manchen Gewebsteilen, z. B. den Epithelien der Haut, annimmt: Nach dieser Richtung, nach weiteren arteriellen Hyperämiemitteln müssen daher unsere Ziele gerichtet sein; deshalb ist es auch naheliegend und geboten, nach weiteren Methoden für arterielle Hy perämie zu suchen.

Zweifellos sind die bekannt gewordenen Beispiele und Versuche für arterielle Hyperämie (die auch Bier anführt), z. B. Arme der Chirurgen usw., bei denen noch thermische und chemische Reize neben der Hyperämie mit in Frage stehen, und die anderen (S. 209-2 Io bei Bi er zitierten) Beispiele, bei denen Nervendurchschneidungen mit von Einfluß auf die sekundären Vorgänge sind, zunächst noch mit etwas Vorsicht als Beispiele für eine arteriell erzeugte Hyperämie zu gebrauchen. Daher hat auch Bier seine Deduktionen noch erst mit Vorsicht gezogen.

Andererseits sind die durch passive Hyperämie klinisch beobachteten und von Bi e r (Seite 190-208) beschriebenen Hypertrophien noch lange nicht im Sinne unserer klinischen Absichten und Wünsche.

Denn diese Hypertrophien betreffen bekanntlich (B i e r Seite 2 I2) nur die Organe mit passiven Funktionen (Stützgewebeund Deckepithelien) (Roux) und gerade die uns wichtigeren Organe mit aktiven Funktionen (Muskeln, Nerven, absondernde Organe usw.) bleiben außer Einfluß. Aber gerade nach der letzteren Richtung liegen, wie gesagt, begreiflicherweise, unsere Wünsche. Daher haben wir logischerweise ein großes Bedürfnis nach weiteren säfte- und blutbewegenden Methoden, namentlich vor allem auch solchen, welche reine arterielle Hyperämie machen, zu suchen, und namentlich vielleicht gerade auch solchen, welche abwechselnd und in kurzen Pausen Anämie und Hyperämie sich folgen lassen. 
Jedenfalls nähern sich diese dann mehr den für ihr Wachstun nötigen physiologischen Bedingungen; denn gerade weil wir seit Virchow wissen, daß eine Zelle sich selbst ernährt, sich nicht passiv ernähren läßt und eine ihr im Überschuß gebotene Nahrung verschmäht, wenn sie nicht gleichzeitig von den in ihrem Wesen uns noch unbekannten Reizen getroffen wird, die sie zum Wachsen und zur Vermehrung bringen (B i e r), müssen solche eine Art künstlichen Stoffwechsel machenden und dic physiologischen Reize nachahmenden Methoden am Platze sein.

Ziehen wir weitere Beobachtungen aus dem täglichen Leben heran: Warum hypertrophiert die Wade der Balleteuse und die Adduktorenmuskulatur des Reiters und der Biceps des Arbeiters: Sind es hier nicht (natürlich neben anderen Momenten) gerade auch die rhythmisch und oft sich wiederholenden Blutentleerungsund Blutfüllungszustände, welche zuletzt dem Muskel zu dem gesteigerten Wachstum verhelfen?

Ich kann diese Frage, die zunächst rein physiologisch ist, an dieser Stelle nicht erschöpfend behandeln. Sie ist eine Aufgabe für eingehende, wissenschaftliche Experimente und Versuche und wäre eines Spezialstudiums wert.

Uns klinische Mediziner aber kann das bis jetzt Gesagte nur zu weiteren klinischen Versuchen ermutigen.

Denn gerade weil die Hochdruckmassage in einer bis jetzt unerreichten Form den oben be. schriebenen Wechsel von Anämie und Hyperämie $\mathrm{macht}$, und eine idealste Form einer Blutmassage darstellt, liegen in ihr große Aussichten und weite Per. spektiven. 